\title{
Effects of retinoic acid-inducible gene-I-like receptors activations and ionizing radiation cotreatment on cytotoxicity against human non-small cell lung cancer in vitro
}

\author{
HIRONORI YOSHINO $^{1}$, MIYU IWABUCHI ${ }^{2}$, YUKA KAZAMA ${ }^{2}$, MAHO FURUKAWA $^{2}$ and IKUO KASHIWAKURA ${ }^{1}$ \\ ${ }^{1}$ Department of Radiation Science, Hirosaki University Graduate School of Health Sciences; \\ ${ }^{2}$ Department of Radiological Technology, Hirosaki University School of Health Sciences, Hirosaki, Aomori 036-8564, Japan
}

Received August 25, 2017; Accepted January 17, 2018

DOI: $10.3892 / \mathrm{ol} .2018 .7867$

\begin{abstract}
Retinoic acid-inducible gene-I (RIG-I)-like receptors (RLRs) are pattern-recognition receptors that recognize pathogen-associated molecular patterns and induce antiviral immune responses. Recent studies have demonstrated that RLR activation induces antitumor immunity and cytotoxicity against different types of cancer, including lung cancer. However a previous report has demonstrated that ionizing radiation exerts a limited effect on RLR in human monocytic cell-derived macrophages, suggesting that RLR agonists may be used as effective immunostimulants during radiation therapy. However, it is unclear whether ionizing radiation affects the cytotoxicity of RLR agonists against cancer cells. Therefore, in the present study the effects of cotreatment with ionizing radiation and RLR agonists on cytotoxicity against human non-small cell lung cancer cells A549 and H1299 was investigated. Treatment with RLR agonist poly(I:C)/LyoVec ${ }^{\mathrm{TM}}$ [poly(I:C)] exerted cytotoxic effects against human non-small cell lung cancer. The cytotoxic effects of poly(I:C) were enhanced by cotreatment with ionizing radiation, and poly(I:C) pretreatment resulted in the radiosensitization of non-small cell lung cancer. Furthermore, cotreatment of A549 and H1299 cells with poly(I:C) and ionizing radiation effectively induced apoptosis in a caspase-dependent manner compared with treatment with poly(I:C) or ionizing radiation alone. These results indicate that RLR agonists and ionizing radiation cotreatment effectively exert cytotoxic effects against human non-small cell lung cancer through caspase-mediated apoptosis.
\end{abstract}

Correspondence to: Dr Hironori Yoshino, Department of Radiation Science, Hirosaki University Graduate School of Health Sciences, 66-1 Hon-cho, Hirosaki, Aomori 036-8564, Japan E-mail: hyoshino@hirosaki-u.ac.jp

Key words: retinoic acid-inducible gene-I-like receptor, ionizing radiation, apoptosis, radiosensitization, caspase, non-small cell lung cancer

\section{Introduction}

Mitochondria are eukaryotic organelles that play a vital role in numerous cellular functions such as oxidative adenosine triphosphate production, calcium homeostasis, and programmed cell death (1). In addition, a recent study showed that mitochondria are involved in innate immune response to RNA viruses (2).

Retinoic acid-inducible gene-I (RIG-I)-like receptors (RLRs) are pattern-recognition receptors (PRRs) that recognize pathogen-associated molecular patterns. RLRs function as cytosolic virus sensors and play an important role in antiviral immunity (3). RLRs include RIG-I, melanoma differentiation-associated gene 5 (MDA5), and laboratory of genetics and physiology 2 (LGP2). RIG-I and MDA5 contain an N-terminal domain consisting of tandem caspase activation and recruitment domains (CARDs), a central DExD/H box RNA helicase domain, and a C-terminal regulatory domain. In contrast, LGP2 lacks CARDs. RIG-I and MDA5 show structural and functional similarities but recognize different RNA viruses (4). RIG-I recognizes relatively short double-stranded RNAs (dsRNAs), 5'-triphosphate single-stranded RNAs, and hepatitis $\mathrm{C}$ viruses. In contrast, MDA5 recognizes long dsRNAs (5) and picornaviruses. Once RIG-I and MDA5 sense an RNA virus invasion, they interact with mitochondrial antiviral-signaling protein (MAVS), an adaptor protein on the mitochondrial membrane, to induce antiviral cytokine type I interferons (IFNs). Therefore, RLRs function as mitochondria-mediated antiviral immune systems.

Recent studies have shown that RLR activation in cancer cells exerts antitumor effects (6-8). Besch et al reported that RIG-I and MDA5 activation in human melanoma cells initiates a proapoptotic signaling pathway in a type I IFN-independent manner (7). They also showed that treatment with RLR ligands exerts antitumor effects in immunodeficient mice, suggesting that RLR activation exerts antitumor effects in the absence of immune activation. Yuan et al reported that treatment with 5'-triphosphate siRNA against the gene encoding vascular endothelial growth factor exerted multiple antitumor effects, including induction of antitumor immunity through RIG-I-mediated innate immune response and induction of 
apoptosis, in non-small cell lung cancer (NSCLC) cells (8). Collectively, these studies indicate that RLRs can be potentially used for treating cancer by inducing antitumor immunity and cytotoxicity against cancer cells.

Radiation is an effective treatment for cancer therapy and is used for treating various cancers such as lung cancers. Our recent study showed that ionizing radiation affected the expression of Toll-like receptors, a type of PRRs, and response to their agonists in human monocytic THP1 cell-derived macrophages (9). However, ionizing radiation exerts a limited effect on RLR expression and response to their agonists in human monocytic THP1 cell-derived macrophages (10). These results suggest that RLR agonists can be used as effective immunostimulants during radiation therapy. However, it is unclear whether ionizing radiation affects the cytotoxicity of RLR agonists.

Lung cancer is the leading cause of cancer-related death over the world, and NSCLC accounts for $85 \%$ of all cases of lung cancer. Since the overall 5-year survival rate of patients with NSCLC remains lower than 15\% (11), development of more effective anticancer strategies is essential for the treatment of NSCLC. Therefore, we investigated the effects of RLR agonists and ionizing radiation cotreatment on cytotoxicity against human NSCLC cells.

\section{Materials and methods}

Reagents. Propidium iodide (PI) and dimethyl sulfoxide were purchased from Sigma-Aldrich (Merck KGaA, Darmstadt, Germany). Z-Val-Ala-Asp (OMe)- $\mathrm{CH}_{2} \mathrm{~F}$ (Z-VAD-fmk) was purchased from Peptide Institute, Inc. (Osaka, Japan). Poly(I:C)-LMW/LyoVec ${ }^{\text {TM }}$ and poly(I:C)-HMW/LyoVec ${ }^{\mathrm{TM}}$ (hereafter referred to as 'poly[I:C]-LMW' and 'poly[I:C]-HMW', respectively) were purchased from InvivoGen (San Diego, CA, USA). Anti-RIG-I antibody (no. 4200), anti-MDA5 antibody (no. 5321), anti-MAVS antibody (no. 3993), anti-caspase-3 antibody (no. 9662), anti- $\beta$-actin antibody (no. 4967), and anti-rabbit horseradish peroxidase (HRP)-linked IgG antibody were purchased from Cell Signaling Technology Japan, K.K. (Tokyo, Japan). Ambion's Silencer ${ }^{\circledR}$ Select Pre-designed siRNA against the gene encoding RIG-I (ID: s24144), Silencer ${ }^{\circledR}$ Select Pre-designed siRNA against the gene encoding MDA5 (ID: s34498), and Silencer ${ }^{\circledR}$ Select Negative Control 1 siRNA were purchased from Thermo Fisher Scientific, Inc. (Waltham, MA, USA).

Cell culture and treatment. Human NSCLC cells A549 and H1299 were purchased from Riken Bio-Resource Center (Tsukuba, Japan) and American Type Culture Collection (ATCC; Manassas, VA, USA), respectively. A549 cells were maintained in Dulbecco's modified Eagle's medium (DMEM; Sigma-Aldrich; Merck KGaA) supplemented with $1 \%$ penicillin/streptomycin $\left(\right.$ Gibco $^{\oplus}$; Gibco; Thermo Fisher Scientific, Inc.) and $10 \%$ heat-inactivated fetal bovine serum (FBS; Japan Bioserum Co., Ltd., Nagoya, Japan) at $37^{\circ} \mathrm{C}$ in a humidified atmosphere of $5 \% \mathrm{CO}_{2}$. $\mathrm{H} 1299$ cells were maintained in RPMI-1640 medium (Gibco ${ }^{\circledR}$; Gibco; Thermo Fisher Scientific, Inc.) supplemented with $1 \%$ penicillin/streptomycin and $10 \%$ heat-inactivated $\mathrm{FBS}$ at $37^{\circ} \mathrm{C}$ in a humidified atmosphere of $5 \% \mathrm{CO}_{2}$.
Cells were seeded in $35-\mathrm{mm}$ culture dishes $\left(6.0 \times 10^{4}\right.$ cells $)$ or 60 -mm culture dishes $\left(1.2 \times 10^{5}\right.$ cells; Iwaki, Chiba, Japan) and were cultured overnight to promote their adherence to the dish. On the next day, the cells were treated with RLR agonist poly(I:C)-LMW or poly(I:C)-HMW (125-1,000 ng/ml) for indicated time periods. Next, the cells were harvested using $0.1 \%$ trypsin-ethylenediaminetetraacetic acid (Gibco ${ }^{\circledR}$; Gibco; Thermo Fisher Scientific, Inc.), and the number of viable cells was counted using trypan blue dye exclusion assay.

In some experiments, the cells were preincubated with $50 \mu \mathrm{M}$ Z-VAD-fmk (a pan-caspase inhibitor) for $1 \mathrm{~h}$, followed by treatment with $250 \mathrm{ng} / \mathrm{ml}$ poly(I:C)-HMW.

Clonogenic survival assay. Cells were seeded in $60-\mathrm{mm}$ culture dishes and were cultured overnight. On the next day, $250 \mathrm{ng} / \mathrm{ml}$ poly(I:C)-HMW were added to the culture medium before $1 \mathrm{~h}$ irradiation, and then exposed to X-ray. After X-ray irradiation, the cells were incubated for $14 \mathrm{~h}$ in the presence of poly(I:C)-HMW. Next, the cells were washed twice with a fresh medium and their culture medium was replaced with a fresh medium. After replacement, the cells were incubated for 8-11 days. Next, the cells were fixed with methanol and were stained with Giemsa solution (Wako Pure Chemical Industries, Ltd., Osaka, Japan). Colonies containing $>50$ cells were counted. The surviving fraction at each dose was calculated with respect to the plating efficiency of the non-irradiated control. The survival curves were fitted to a linear-quadratic model: $\mathrm{SF}=\exp \left(-\alpha D-\beta D^{2}\right)$, where $\mathrm{SF}$ is the surviving fraction and $D$ is the physical dose, by data analysis software Origin Pro 9.0J (OriginLab Co., Northampton, MA, USA).

siRNA transfection. A549 cells were transfected with target siRNAs or control siRNA by using Lipofectamine RNAiMAX (Invitrogen; Thermo Fisher Scientific, Inc.), according to the manufacturer's instructions. The final concentration of the siRNAs was $10 \mathrm{nM}$. After incubation for $24 \mathrm{~h}$, the transfected cells were harvested and were used for performing subsequent analyses.

In vitro irradiation. The cells were irradiated $(150 \mathrm{kVp}$, $20 \mathrm{~mA}, 0.5-\mathrm{mm} \mathrm{Al} \mathrm{filter,} \mathrm{and} 0.3$-mm Cu filter) by using an X-ray generator (MBR-1520R-3; Hitachi Medical Corporation, Tokyo, Japan) at a distance of $45 \mathrm{~cm}$ from the focus and at a dose rate of 1.00-1.04 Gy/min.

Sodium dodecyl sulfate-polyacrylamide gel electrophoresis and western blotting. Harvested cells were lysed in 1X Laemmli sample buffer (Bio-Rad Laboratories, Inc., Hercules, CA, USA) containing 2.5\% 2-mercaptoethanol through sonication, and the obtained cell lysates were boiled for $10 \mathrm{~min}$. Protein concentration of the cell lysates was determined using XL-Bradford assay kit (APRO Science, Tokushima, Japan) and SmartSpec ${ }^{\mathrm{TM}}$ plus spectrophotometer (Bio-Rad Laboratories, Inc.). Sodium dodecyl sulfate-polyacrylamide gel electrophoresis and western blotting were performed, as reported previously (12). The following primary antibodies were used: anti-RIG-I antibody (dilution, 1:3,000), anti-MDA5 antibody (dilution, 1:3,000), anti-MAVS antibody (dilution, 1:3,000), anti-caspase-3 antibody (dilution, 1:3,000), 
and anti-actin antibody (dilution, 1:4,000). The following secondary antibody was used: HRP-linked anti-rabbit IgG antibody (dilution, 1:10,000). Antigens were visualized using ECL Prime Western Blotting Detection System (GE Healthcare UK Ltd., Buckinghamshire, UK). Blots were stripped using a Stripping Solution (Wako Pure Chemical Industries, Ltd.).

Analysis of cell death. Cell death was analyzed using Annexin V-FITC (BioLegend Inc., San Diego, CA, USA), PI, and Annexin $\mathrm{V}$ binding buffer (BioLegend Inc.), as reported previously (13). Stained cells were analyzed by performing flow cytometry (Cytomics FC500; Beckman Coulter, Inc., Brea, CA, USA). In the Annexin V/PI quadrant gating, Annexin $\mathrm{V}^{-} / \mathrm{PI}^{-}$, Annexin $\mathrm{V}^{+} / \mathrm{PI}^{-}$, and Annexin $\mathrm{V}^{+} / \mathrm{PI}^{+}$were used to identify the fraction of viable cells, early apoptotic cells, and late apoptotic/necrotic cells, respectively.

Detection of $\gamma-H 2 A X$ by performing flow cytometry. Harvested cells were fixed overnight in ice-cold $70 \%$ methanol at $-20^{\circ} \mathrm{C}$. The fixed cells were washed using a wash buffer (WB; PBS containing $0.5 \%$ bovine serum albumin) and were treated with a WB containing $0.25 \%$ Triton $\mathrm{X}-100$ on ice for $5 \mathrm{~min}$. After washing with the $\mathrm{WB}$, cell pellets were incubated for $1 \mathrm{~h}$ at room temperature with anti-phosphorylated histone $\mathrm{H} 2 \mathrm{AX}$ monoclonal antibody (JBW301; Upstate Biotechnology, Lake Placid, NY, USA) diluted to 300-folds by using the WB containing $0.25 \%$ Triton $\mathrm{X}-100$. The labeled cells were washed with the WB and were treated in the dark for $1 \mathrm{~h}$ at room temperature with Alexa Fluor $488^{\circledR}$-conjugated anti-mouse IgG secondary antibody (Molecular Probes; Thermo Fisher Scientific, Inc.) diluted to 400 -folds by using the WB containing $0.25 \%$ Triton X-100. The stained cells were washed with the WB and were analyzed by performing flow cytometry.

Statistical analysis. Data are presented as mean \pm standard error (SE). Comparisons between control and experimental groups were performed using a two-sided Student's t-test or a two-sided Mann-Whitney's U-test depending on the data distribution. Multiple data were analyzed using one-factor analysis of variance followed by Tukey-Kramer test. Differences were considered significant at $\mathrm{P}<0.05$. All statistical analyses were performed using Excel 2010 software (Microsoft Corporation, Redmond, WA, USA) with an add-in software Statcel 3 (The Publisher OMS Ltd., Tokyo, Japan).

\section{Results}

RLR agonists exert cytotoxicity against NSCLC cells. We first examined the cytotoxicity of RLR agonists against A549 and H1299 cells. In the present study, poly(I:C)-LyoVec ${ }^{\mathrm{TM}}$, a complex of a synthetic dsRNA analogue poly(I:C) and transfection reagent $\mathrm{LyoVec}^{\mathrm{TM}}$, was used as an RLR agonist. RIG-I and MDA5 expression was undetectable or negligible in untreated A549 and H1299 cells and strongly increased in RLR agonists poly(I:C)-LMW- and poly(I:C)-HMW-treated A549 and H1299 cells (Fig. 1A). Moreover, poly(I:C)-LMW and poly(I:C)-HMW treatment significantly decreased the number of viable cells and increased the percentages of Annexin $\mathrm{V}^{+}$ cells in a dose-dependent manner (Fig. 1B and C, respectively). Because poly(I:C)-HMW exerted a stronger cytotoxic effect than poly(I:C)-LMW, we used poly(I:C)-HMW in all subsequent experiments.

Effects of the RLR agonist and ionizing radiation cotreatment on cytotoxicity against NSCLC cells. We next examined the effect of the RLR agonist and ionizing radiation cotreatment on cytotoxicity against NSCLC cells. Cotreatment with poly(I:C)-HMW and X-ray irradiation ( 2 and $4 \mathrm{~Gy}$ ) significantly decreased the number of viable cells compared with treatment with poly(I:C)-HMW or X-ray irradiation alone (Fig. 2A). Furthermore, cotreatment with poly(I:C)-HMW and 4 Gy $\mathrm{X}$-ray irradiation increased the percentages of Annexin $\mathrm{V}^{+}$cells compared with treatment with poly(I:C)-HMW or X-ray irradiation alone (Fig. 2B). These results indicate that the RLR agonist and ionizing radiation cotreatment effectively induced the cytotoxicity against NSCLC cells.

Radiosensitizing effects of the RLR agonist on NSCLC cells. We next examined the radiosensitizing effects of poly(I:C)-HMW on NSCLC cells. Clonogenic cell survival decreased in irradiated A549 and H1299 cells pretreated with poly(I:C)-HMW (Fig. 3A). As shown in Table I, the radiation dose that $37 \%$ of cells will survival $\left(\mathrm{D}_{37}\right)$ was reduced from $3.02 \mathrm{~Gy}$ in the control to $2.35 \mathrm{~Gy}$ by the treatment with poly(I:C)-HMW in A549 cell $(\mathrm{P}<0.01)$. Similarly, the $\mathrm{D}_{37}$ was reduced from $3.47 \mathrm{~Gy}$ in the control to 2.46 Gy by the treatment with poly(I:C)-HMW in $\mathrm{H} 1299$ cells $(\mathrm{P}<0.05)$. The sensitizer enhancement ratio (SER) judged by the $\mathrm{D}_{37}$ in the A549 and H1299 cells were 1.28 and 1.41 , respectively. These results suggest that the RLR agonist exerted radiosensitizing effects on NSCLC cells.

To investigate mechanisms underlying the radiosensitizing effects of poly(I:C)-HMW, we analyzed the repair of DNA double-stranded breaks (DSBs). Histone H2AX undergoes phosphorylation at serine $139(\gamma$-H2AX) immediately after DSB induction and undergoes dephosphorylation after DSB repair (14). Therefore, we analyzed $\gamma$-H2AX expression in NSCLC cells treated with 4 Gy X-ray irradiation and/or poly(I:C)-HMW. Treatment with the ionizing irradiation increased $\gamma-\mathrm{H} 2 \mathrm{AX}$ expression in A549 cells irrespective of poly(I:C)-HMW treatment (Fig. 3B). No significant difference was observed in $\gamma$-H2AX expression between cells treated with $4 \mathrm{~Gy} X$-ray irradiation alone and cells treated with poly(I:C)-HMW + 4 Gy X-ray irradiation at 1 and $3 \mathrm{~h}$ after the irradiation. However, $\gamma-\mathrm{H} 2 \mathrm{AX}$ expression was higher in cells treated with poly(I:C)-HMW + 4 Gy X-ray irradiation than in cells treated with 4 Gy X-ray irradiation alone at $24 \mathrm{~h}$ after the irradiation. Similarly, $\gamma-\mathrm{H} 2 \mathrm{AX}$ expression was higher in H1299 cells treated with poly(I:C)-HMW + 4 Gy X-ray irradiation than in cells treated with $4 \mathrm{~Gy} \mathrm{X}$-ray irradiation alone at $48 \mathrm{~h}$ after the irradiation (Fig. 3C). These results suggest that poly(I:C)-HMW inhibits DSB repair in irradiated cells, which leads to their radiosensitization.

Effects of the RLR agonist and ionizing radiation cotreatment on RLR and MAVS expression. We next explored mechanisms underlying the effect of poly(I:C)-HMW and ionizing radiation cotreatment on cell death induction in A549 and H1299 cells. Knockdown of RIG-I and MDA5 in A549 cells decreased 
A $\mathbf{A 5 4 9}$

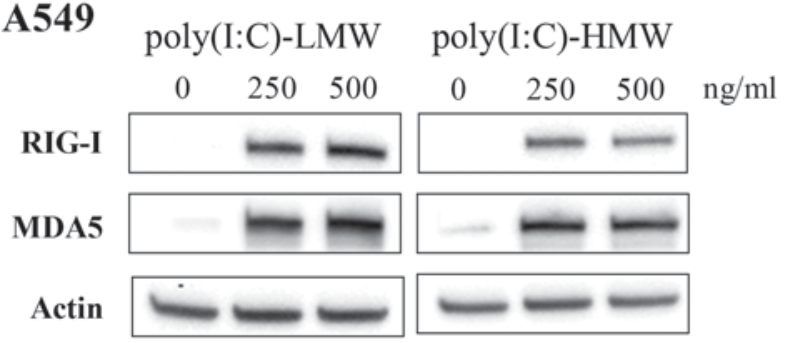

H1299

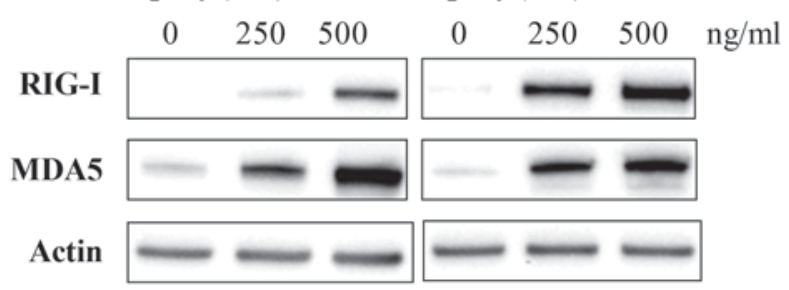

$\mathrm{B}$
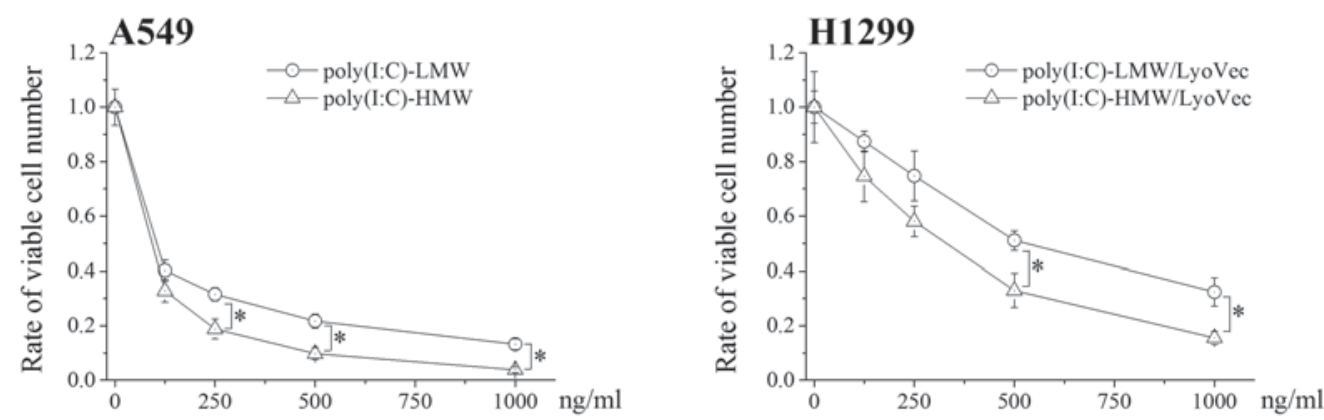

$\mathrm{C}$
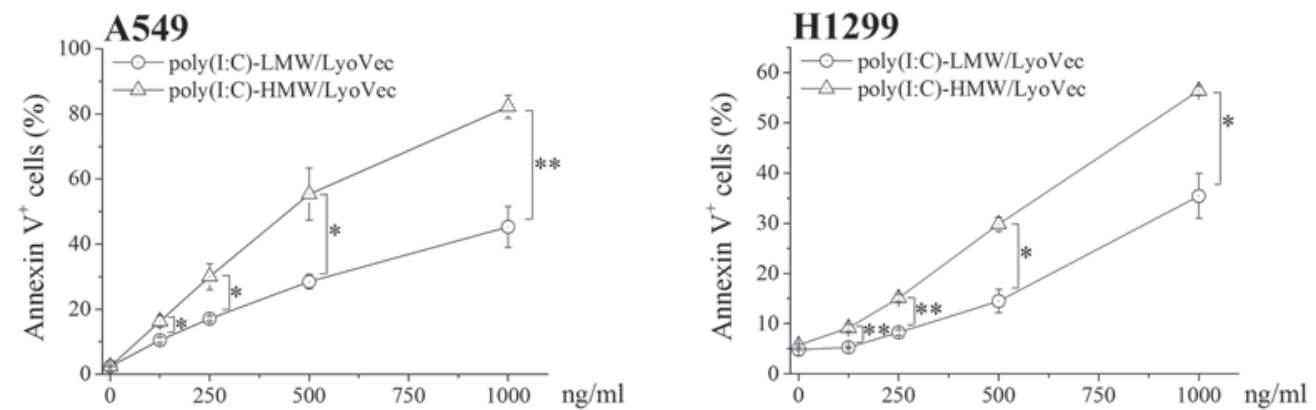

Figure 1. Effects of retinoic acid-inducible gene-I (RIG-I)-like receptor agonists on the growth of and apoptosis induction in non-small cell lung cancer cells. (A) A549 and H1299 cells were treated with poly(I:C)-LMW and poly(I:C)-HMW for 24 (for A549) or 48 h (for H1299) and were harvested for performing western blotting analyses of RIG-I and MDA5; actin was used as a loading control. Representative blots are shown. (B and C) A549 and H1299 cells cultured with the indicated concentrations of poly(I:C)-LMW or poly(I:C)-HMW for 3 days were harvested, and the number of viable cells was counted by performing the trypan blue exclusion assay (B). Annexin V/PI staining was performed to evaluate the cell death (C). Data are presented as mean \pm SE of at least 3 independent experiments; ${ }^{\mathrm{P}}<0.05$ and ${ }^{* *} \mathrm{P}<0.01$.

poly(I:C)-HMW-induced RIG-I and MDA5 expression (Fig. 4A) and significantly decreased poly(I:C)-HMW-induced Annexin $\mathrm{V}^{+}$cells (Fig. 4B). Because these results suggested that RLRs mediated poly(I:C)-HMW-induced cell death, we hypothesized that the effects of poly(I:C)-HMW and ionizing radiation cotreatment on cell death induction were induced by the upregulation of RLR expression. However, ionizing radiation did not increase poly(I:C)-HMW-induced increase in RIG-I and MDA5 expression (Fig. 4C). Next, we analyzed the protein expression of MAVS, which functions as an adaptor protein for RLR-mediated signaling pathways (15). It is reported that proteasome-mediated MAVS degradation occurs after RLR activation, and this degradation is required for downstream signaling leading to type I IFN production (16). Consistently, we observed that poly(I:C)-HMW treatment downregulated MAVS protein expression in A549 cells. However, no significant difference in MAVS protein expression was observed between cells treated with poly(I:C)-HMW alone and cells treated with poly(I:C)-HMW + 4 Gy X-ray irradiation (Fig. 4C).
The RLR agonist and ionizing radiation cotreatment effectively induce apoptosis by activating caspase. Caspases are involved in RLR-induced apoptosis (7). As shown in Fig. 5A, poly(I:C)-HMW induced active caspase-3 expression in A549 and H1299 cells (Fig. 5A). Furthermore, treatment with the pan-caspase inhibitor Z-VAD-fmk significantly decreased poly(I:C)-HMW-induced Annexin $\mathrm{V}^{+} / \mathrm{PI}^{-}$early apoptotic cells in A549 cells (Fig. 5B). Similarly, Z-VAD-fmk tended to decrease Annexin $\mathrm{V}^{+} / \mathrm{PI}^{-}$early apoptotic cells in $\mathrm{H} 1299$ cells $(\mathrm{P}=0.052)$. These results indicate that RLR agonists induce apoptosis in human NSCLC cells by activating caspases. Next, we investigated the involvement of caspases in the effects of the RLR agonist and ionizing radiation cotreatment. Cotreatment with poly(I:C)-HMW and 4 Gy $\mathrm{X}$-ray irradiation increased active caspase-3 expression compared with treatment with poly(I:C)-HMW or 4 Gy X-ray irradiation alone (Fig. 5A). Furthermore, the percentages of Annexin $\mathrm{V}^{+} / \mathrm{PI}^{-}$early apoptotic cells in A549 and H1299 cells were significantly decreased by treatment with Z-VAD-fmk (Fig. 5B and C). The percentages of Annexin $\mathrm{V}^{+} / \mathrm{PI}^{+}$late 
A

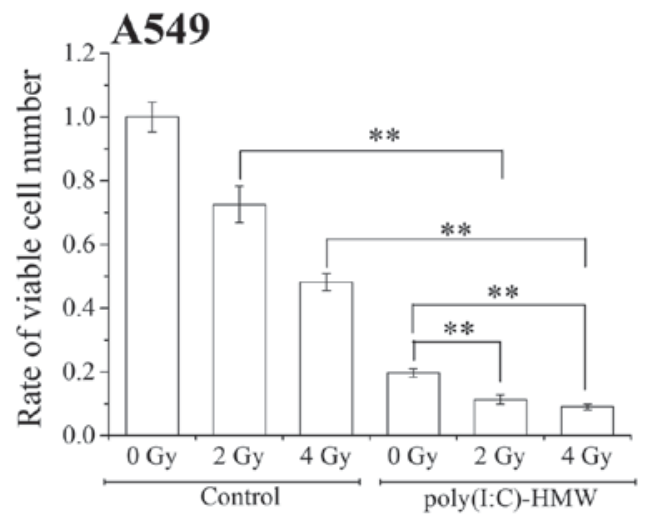

B

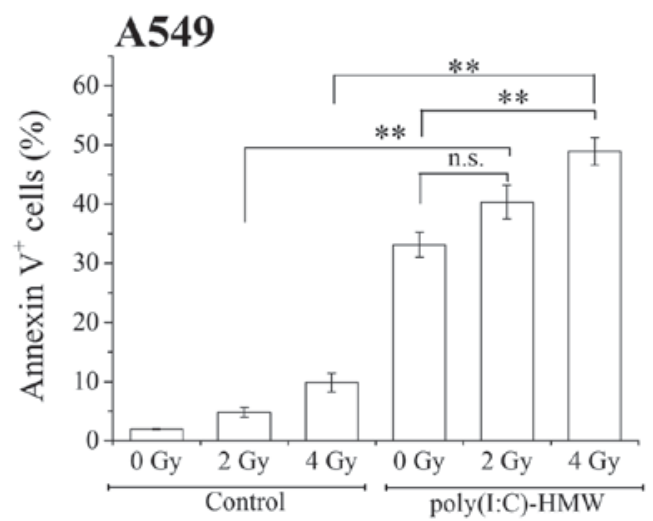

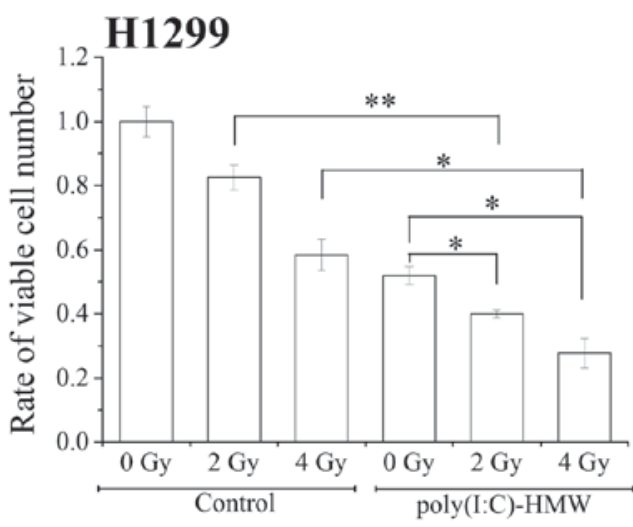

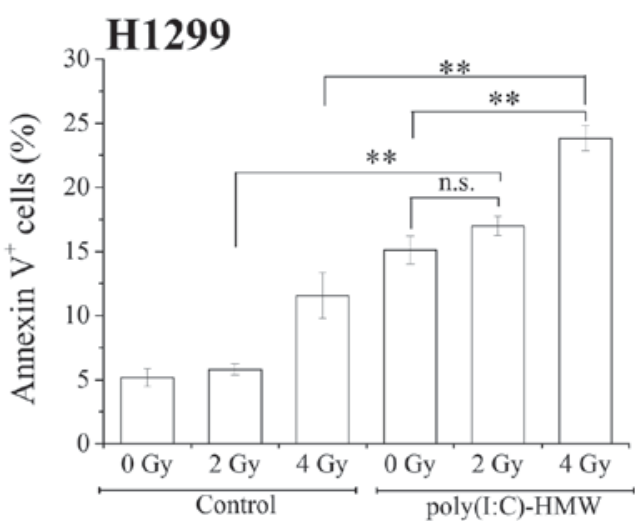

Figure 2. Effects of cotreatment with the retinoic acid-inducible gene-I (RIG-I)-like receptor agonist and ionizing radiation on the growth of and cell death induction in non-small cell lung cancer cells. (A and B) Cells were preincubated with poly(I:C)-HMW for $1 \mathrm{~h}$, and X-ray irradiation was performed. The cells were harvested after culturing for 3 days. The number of viable cells was determined by performing the trypan blue exclusion assay, and the cell death was determined by performing Annexin V/PI staining. Data are presented as the mean $\pm \mathrm{SE}$ of at least 3 independent experiments; $\mathrm{P}<0.05$ and ${ }^{* *} \mathrm{P}<0.01$; $\mathrm{n} . \mathrm{S}$., not significant.

apoptotic cells/necrotic cells in H1299 cells, not A549 cells, were also significantly decreased by treatment with Z-VAD-fmk (Fig. 5C). Together, these results suggest that the effects of the RLR agonist and ionizing radiation cotreatment on cell death induction including apoptosis are mediated by caspase activation.

\section{Discussion}

We previously investigated the effect of ionizing radiation on PRRs in human monocytic cells $(9,10)$ and showed that ionizing radiation negligibly affected RLR expression and response to their agonists (10), suggesting that RLR agonists could be used as effective immunostimulants during radiation therapy. In the present study, we investigated the effects of RLR agonists and ionizing radiation cotreatment on cytotoxicity against NSCLC cells. We found that cotreatment with poly(I:C)-HMW and ionizing radiation effectively suppressed the growth of and induced cell death in NSCLC cells. Furthermore, we found that poly(I:C)-HMW treatment exerted radiosensitizing effects on NSCLC cells probably by affecting DSB repair capacity. Although we did not determine mechanisms underlying the attenuation of DSB repair by the activation of mitochondria-mediated immune systems in the present study, our results showed that cotreatment with the RLR agonist and ionizing radiation is a promising strategy to enhance cytotoxicity against NSCLC cells.
We found that poly(I:C)-HMW exerted a stronger cytotoxic effect than poly(I:C)-LMW against NSCLC cells. The difference between poly(I:C)-LMW and poly(I:C)-HMW is a molecular weight. According to the manufacture's data sheet, the average size of poly(I:C)-LMW and poly(I:C)-HMW is $0.2-1$ and $1.5-8 \mathrm{~kb}$, respectively. It is thought that poly(I:C)-LMW and poly(I:C)-HMW are mainly recognized by RIG-I and MDA5, respectively, because RIG-I and MDA5 preferentially recognizes short $(\sim 0.3 \mathrm{~kb})$ and long (>4 kb) poly(I:C), respectively (4). These imply that MDA5 may be a better target for inducing cytotoxicity than RIG-I. However, knockdown of RIG-I effectively suppressed poly(I:C)-HMW-induced cell death compared with knockdown of MDA5 (Fig. 4B), thus suggesting that not only MDA5 but also RIG-I are involved in the poly(I:C)-HMW induced cell death. Interestingly, we found that knockdown of RIG-I downregulated poly(I:C)-HMW-induced MDA5 expression as well as RIG-I expression (Fig. 4A). Consistently, Imaizumi et al reported that poly(I:C) transfection induced MDA5 expression through RIG-I and IFN- $\beta$ (17). Therefore, it is possible that RIG-I mediates the poly(I:C)-HMW-induced MDA5 expression, which contributes to the poly(I:C)-HMW-induced cell death.

There are some researches to improve the RLR-mediated antitumor activity. For example, some researcher focused on the recognition of 5'-triphosphate single-stranded RNAs by RIG-I, and designed the 5'-triphosphate-siRNA $(8,18,19)$. 

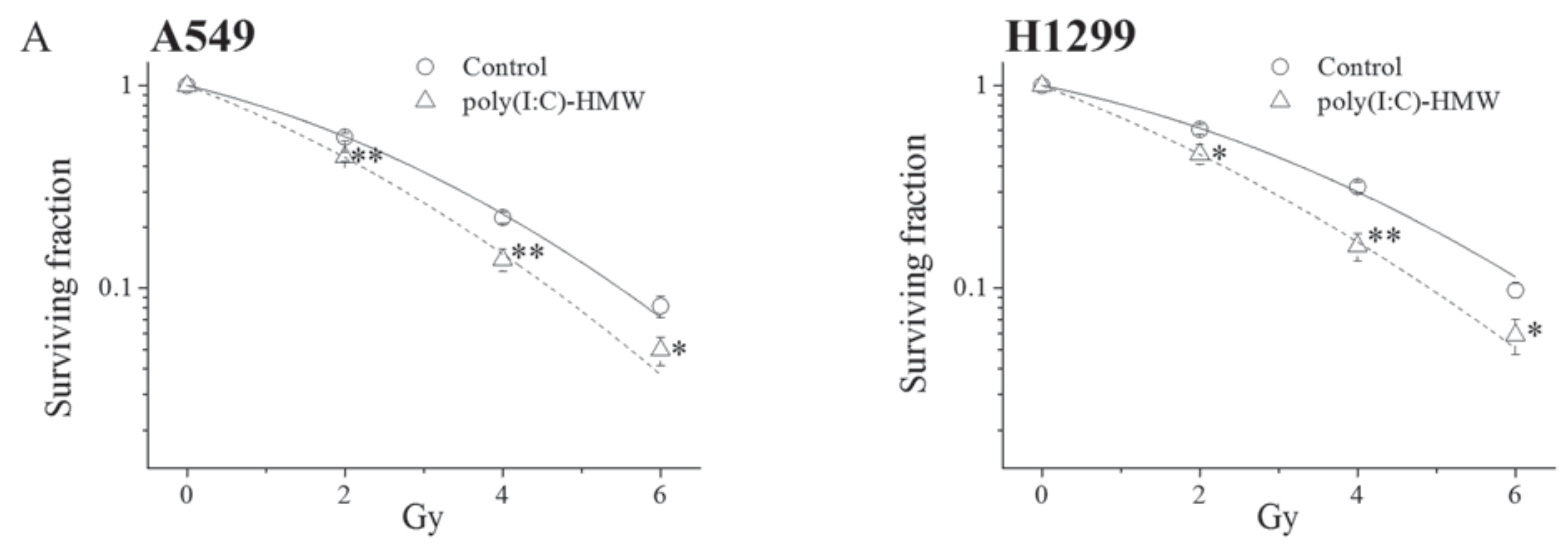

В $\mathbf{A 5 4 9}$
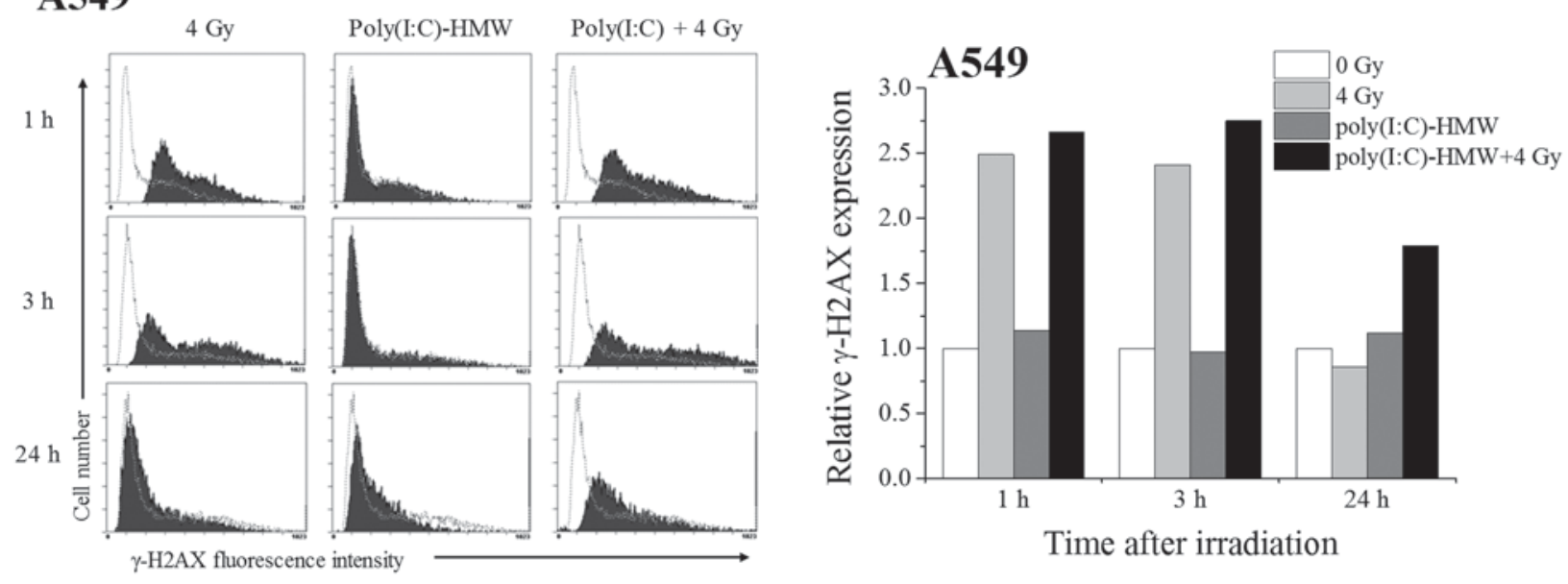

\section{C $\mathbf{H 1 2 9 9}$}
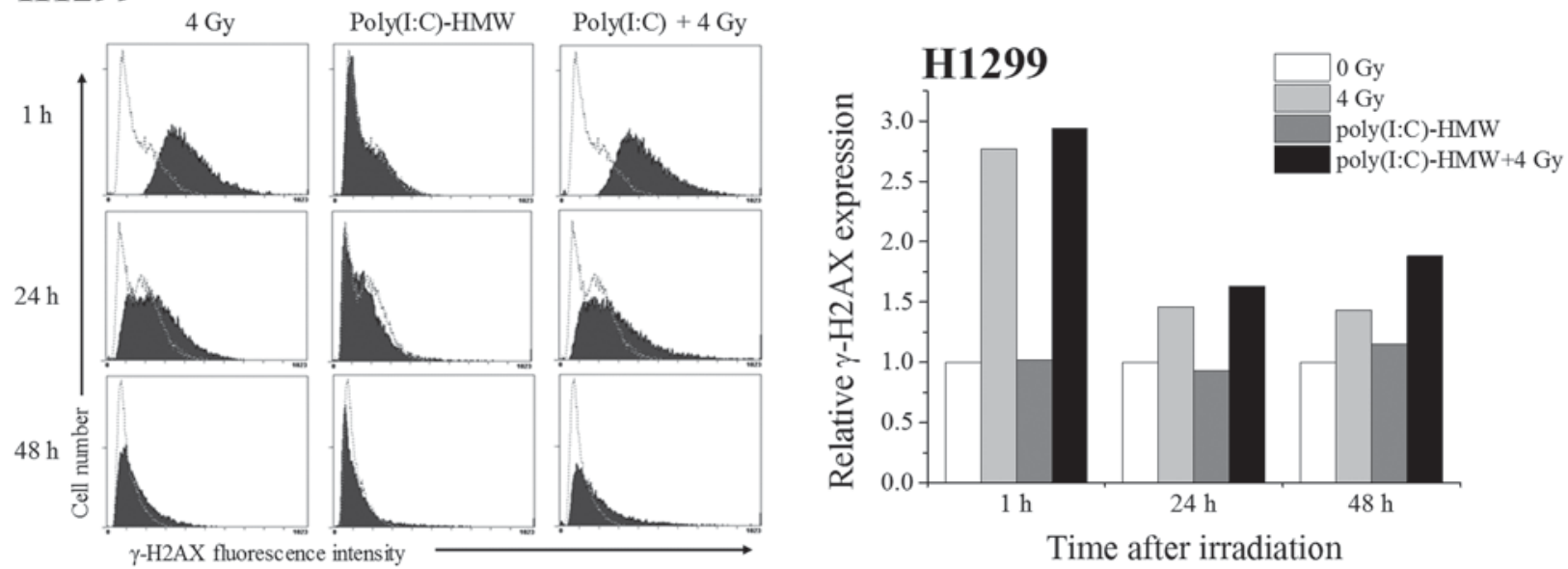

Figure 3. Effects of the retinoic acid-inducible gene-I (RIG-I)-like receptor agonist on the radiosensitivity of and $\gamma$-H2AX expression in non-small cell lung cancer cells. (A) Cellular radiosensitivity was analyzed by performing the clonogenic survival assay, as described in Materials and Methods. Data are presented as the mean \pm SE of 3 independent experiments performed in triplicate; ${ }^{*} \mathrm{P}<0.05$ and ${ }^{* * *} \mathrm{P}<0.01$ compared with control cells. (B and C) A549 (B) and H1299 cells (C) were pretreated with poly(I:C)-HMW for $1 \mathrm{~h}$, followed by treatment with $4 \mathrm{~Gy}$ X-ray irradiation. Cells were harvested at the indicated time points, and $\gamma$-H2AX expression was analyzed. Representative histograms of $\gamma$-H2AX expression are shown. The dotted line histogram indicates the data of non-irradiated and non-treated with poly(I:C)-HMW, and filled black histograms indicates the 4 Gy-irradiated and/or poly(I:C)-HMW-treated cells. The relative value of median fluorescence intensity of $\gamma$-H2AX compared with that for non-irradiated and non-treated with poly(I:C)-HMW control cells re shown in the right bar graph. Representative data of similar results from two independent experiments are shown.

Yuan et al designed a 5'-triphosphate-siRNA targeting vascular endothelial growth factor (VEGF). The 5'-triphosphate-siRNA targeting VEGF showed multiple antitumor effects against NSCLC through not only induction of RIG-mediated apoptosis and antitumor immunity but also inhibition of tumor angiogenesis by knockdown of VEGF (8). On the other hand, Duewell et al reported a cotreatment with RLR agonists and an activating monoclonal antibody for death receptor Fas on cytotoxicity. In their report, it was demonstrated that RLR agonists increased the cell surface Fas expression, which 
Table I. Summary of survival curve parameters.

\begin{tabular}{llcccc}
\hline Cell & \multicolumn{1}{c}{ Treatment } & $\alpha\left(\mathrm{Gy}^{-1}\right)$ & $\beta\left(\mathrm{Gy}^{-2}\right)$ & $\mathrm{D}_{37}(\mathrm{~Gy})$ & SER $\left(\mathrm{D}_{37}\right)$ \\
\hline A549 & Control & 0.216 & 0.037 & 3.02 & 1.28 \\
& Poly(I:C)-HMW & 0.341 & 0.034 & 2.35 & 1.41 \\
\multirow{2}{*}{ H1299 } & Control & 0.182 & 0.030 & 3.47 & 2.46 \\
& Poly(I:C)-HMW & 0.339 & 0.026 & 1.41 \\
\hline
\end{tabular}

$D_{37}$, the radiation dose at which $37 \%$ of cells will survival; SER, sensitizer enhancement ratio.

A

poly(I:C)-HMW $0 \mathrm{ng} / \mathrm{ml}$ poly(I:C)-HMW $250 \mathrm{ng} / \mathrm{ml}$

siRNA Control RIG-I MDA5 Control RIG-I MDA5

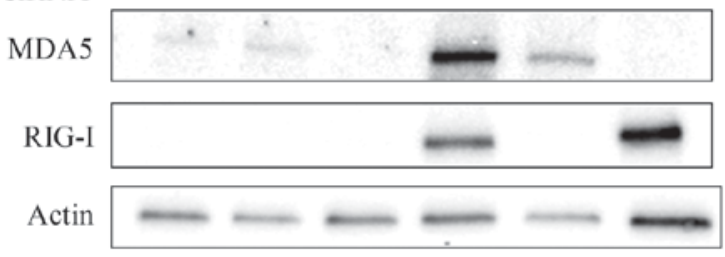

B

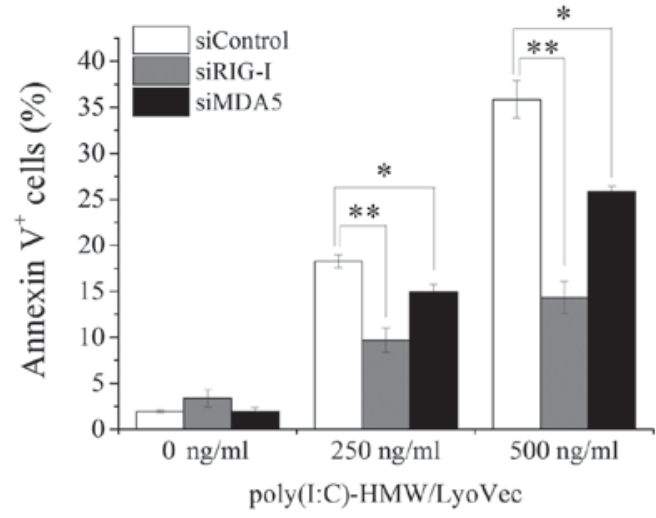

$72 \mathrm{~h}$

C

$48 \mathrm{~h}$

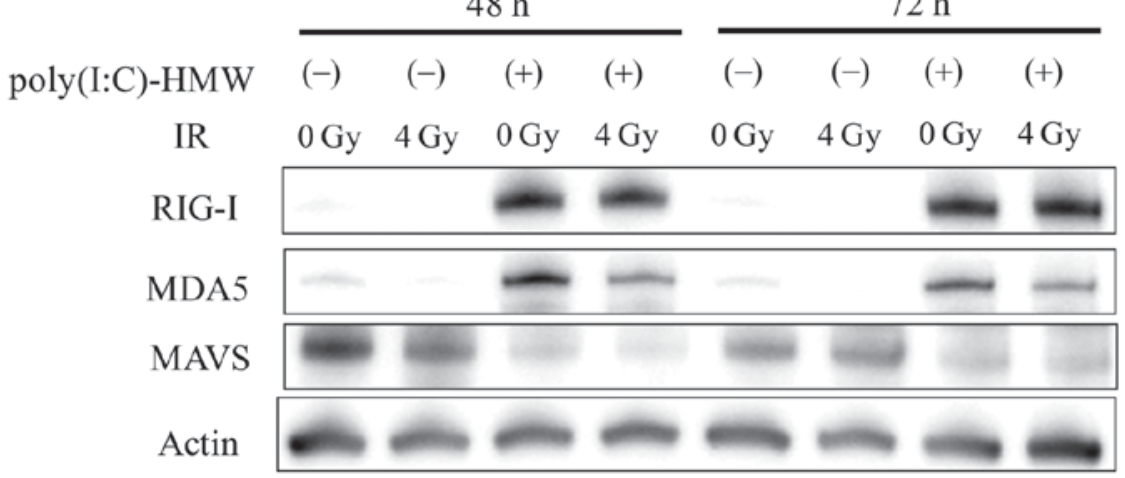

Figure 4. Involvement of retinoic acid-inducible gene-I (RIG-I)-like receptor (RLR) expression in RLR agonist-induced cell death of A549 cells. (A) A549 cells treated with the siRNA against RIG-I or melanoma differentiation-associated gene 5 (MDA5) were cultured with 250 ng/ml poly(I:C)-HMW for $24 \mathrm{~h}$ and were harvested for performing western blotting of RIG-I and MDA5; actin was used as a loading control. Representative blots are shown. (B) A549 cells treated with the siRNA against the gene encoding RIG-I or MDA5 were cultured with $250 \mathrm{ng} / \mathrm{ml}$ poly(I:C)-HMW for $48 \mathrm{~h}$ and were harvested for performing Annexin V/PI staining. Percentages of Annexin $\mathrm{V}^{+}$cells are presented as the mean $\pm \mathrm{SE}$ of 3 independent experiments; ${ }^{*} \mathrm{P}<0.05$ and ${ }^{* *} \mathrm{P}<0.01$. (C) A549 cells were preincubated with poly(I:C)-HMW for $1 \mathrm{~h}$ and were irradiated with $4 \mathrm{~Gy}$ X-rays. The cells were harvested at the indicated time points, and western blotting of RIG-I, MDA5, and mitochondrial antiviral-signaling (MAVS) was performed; actin was used as a loading control. Representative blots are shown.

sensitized pancreatic cancer cells towards Fas-mediated cell killing (20). In the present study, we focused on the ionizing radiation to improve RLR agonist-induced cytotoxicity, and showed that cotreatment with RLR agonist and ionizing radiation effectively induced the cytotoxicity against cancer cells for the first time.

Recently, Ranoa et al showed that depletion of RIG-I protected mice from death following to total body irradiation (21). They also showed that ionizing radiation increased the RIG-I expression, not MDA5 expression, and that RIG-I recognize small endogenous noncoding RNA induced by ionizing radiation, which resulted in the activation of IFN signaling through MAVS. Considering that we did not observe the enhancement of RIG-I and MDA5 expression by 4 Gy $\mathrm{X}$-ray irradiation in the presence or absence and poly(I:C)-HMW (Fig. 4C), there is a possibility that the poly(I:C)-HMW-induced RIG-I recognizes small endogenous noncoding RNA induced by ionizing radiation, which effectively increases the poly(I:C)-HMW-induced cell death. However, we need to investigate the RLR/MAVS-mediated signaling pathway in the cells cotreated with RLR agonist and ionizing radiation in detail in a future study, because the difference in the MAVS expression between poly(I:C)-HMW and poly(I:C)-HMW + 4 Gy X-ray irradiation was not observed in the present study (Fig. 4C).

RLR activation induces type I IFN. The type I IFN such as IFN- $\beta$ is known to improve the response of tumor to ionizing radiation (22-24). For example, type I IFN affects 
A

A549

H1299

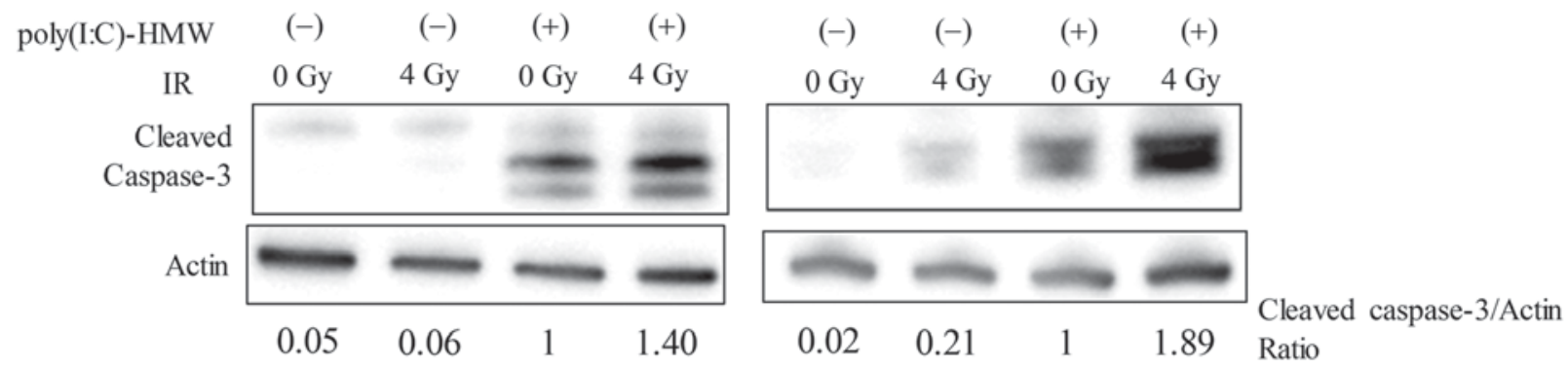

B
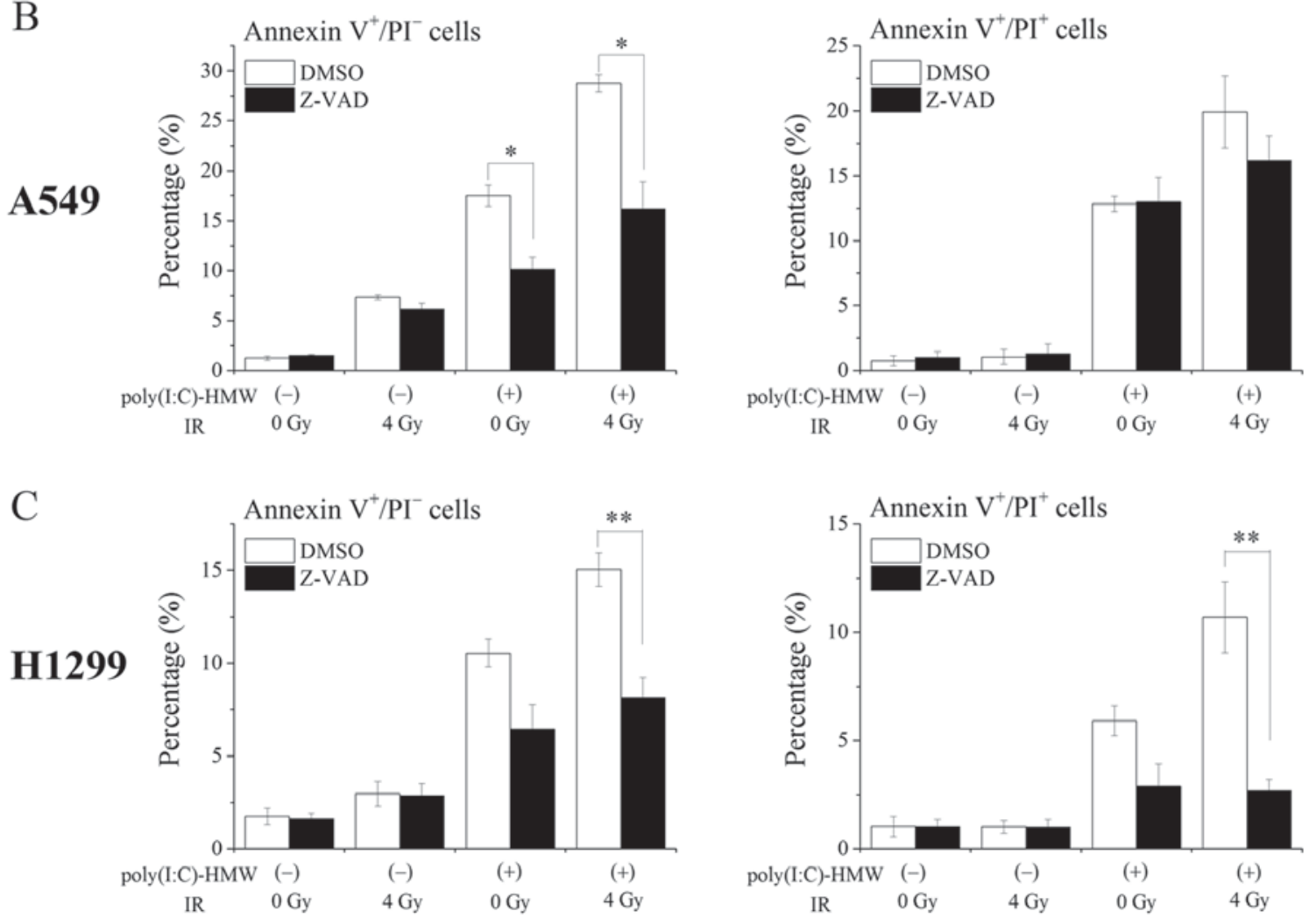

Figure 5. Involvement of caspase in the effects of the retinoic acid-inducible gene-I (RIG-I)-like receptor (RLR) agonist and ionizing radiation cotreatment on apoptosis induction in non-small cell lung cancer cells. (A) Cells were preincubated with poly(I:C)-HMW for 1 h, followed by irradiation with 4 Gy X-rays. After culturing for 3 days, the cells were harvested for performing western blotting of caspase-3; actin was used as a loading control. Representative blots are shown. The quantification of bands was performed using ImageJ software, and the relative values of cleaved caspase-3/actin ratio compared with that for non-irradiated and treated with poly(I:C)-HMW control are shown. (B and C) A549 (B) and H1299 cells (C) were preincubated with Z-VAD-fmk for $1 \mathrm{~h}$, followed by treatment with $250 \mathrm{ng} / \mathrm{ml}$ poly(I:C)-HMW. After incubation for $1 \mathrm{~h}$, the cells were irradiated with $4 \mathrm{~Gy}$ X-rays. After culturing for $72 \mathrm{~h}$, the cells were harvested for performing Annexin V/PI staining. The percentages of Annexin $\mathrm{V}^{+} / \mathrm{PI}^{-}$cells and Annexin $\mathrm{V}^{+} / \mathrm{PI}^{+}$cells were shown. Data are presented as the mean \pm SE of 3 independent experiments; ${ }^{*} \mathrm{P}<0.05$ and ${ }^{* *} \mathrm{P}<0.01$. DMSO, dimethyl sulfoxide; Z-VAD, Z-VAD-fmk.

the efficacy of radiotherapy through activation of immune cells (23). Furthermore, the radiosensitizing effects of exogenous IFN- $\beta$ was reported (24). Therefore, it is possible that the radiosensitizing effect of poly(I:C)-HMW and the effects of poly(I:C)-HMW and ionizing radiation cotreatment on cytotoxicity are due to the induction of type I IFN. However, because it is reported that RLR/MAVS signaling pathway induces apoptosis in a IFN-independent manner $(7,25,26)$, we need a further study to clarify whether the effects of RLR agonist and ionizing radiation cotreatment on cytotoxicity depends on the type I IFN.

Results of the present study suggested the involvement of caspase activation in the effects of the RLR agonist and ionizing radiation cotreatment on cytotoxicity against NSCLC cells. It is reported that RLR activation induces apoptosis via intrinsic (caspase-9) and/or extrinsic (caspase-8) apoptotic pathway. Besch et al reported that caspase-9 plays important roles in RLR-induced apoptosis in human melanoma cells (7). On the other hand, the activation of MDA5 by Semliki Forest virus induces both caspase-9-mediated apoptosis and caspase-8-mediated extrinsic apoptosis (27). Since ionizing radiation can modulate the intrinsic and extrinsic apoptotic pathway (28-31), further studies are needed to clarify the apoptotic pathway which RLR agonist and ionizing radiation cooperatively activate.

In conclusion, results of the present study showed that cotreatment with ionizing radiation and the RLR agonist effectively induced cytotoxicity against human NSCLC cells, 
suggesting that RLR activations effectively enhanced the apoptosis and radiosensitivity of cancer cells and improved antitumor immunity during radiation therapy. However, because treatment with poly(I:C)-LMW $(1 \mu \mathrm{g} / \mathrm{ml})$ induces apoptosis in human hematopoietic stem/progenitor cells (32), further studies are needed to investigate the effects of cotreatment with the RLR agonist and ionizing radiation on cytotoxicity against normal cells and to determine a strategy for minimizing its side effects.

\section{Acknowledgements}

This study was supported by JSPS KAKENHI (grant no. JP25861053) and was partially supported by JSPS KAKENHI (grant no. JP15K09985) and Takeda Science Foundation [HY].

\section{References}

1. Duchen MR: Mitochondria in health and disease: Perspectives on a new mitochondrial biology. Mol Aspects Med 25: 365-451, 2004.

2. Pourcelot M and Arnoult D: Mitochondrial dynamics and the innate antiviral immune response. FEBS J 281: 3791-3802, 2014

3. Matsumiya T and Stafforini DM: Function and regulation of retinoic acid-inducible gene-I. Crit Rev Immunol 30: 489-513, 2010.

4. Kato H, Takeuchi O, Mikamo-Satoh E, Hirai R, Kawai T, Matsushita K, Hiiragi A, Dermody TS, Fujita T and Akira S: Length-dependent recognition of double-stranded ribonucleic acids by retinoic acid-inducible gene-I and melanoma differentiation-associated gene 5. J Exp Med 205: 1601-1610, 2008.

5. Kato H, Takeuchi O, Sato S, Yoneyama M, Yamamoto M, Matsui K, Uematsu S, Jung A, Kawai T, Ishii KJ, et al: Differential roles of MDA5 and RIG-I helicases in the recognition of RNA viruses. Nature 441: 101-105, 2006.

6. Li K, Qu S, Chen X, Wu Q and Shi M: Promising targets for cancer immunotherapy: TLRs, RLRs, and STING-mediated innate immune pathways. Int J Mol Sci 18: pii: E404, 2017.

7. Besch R, Poeck H, Hohenauer T, Senft D, Häcker G, Berking C, Hornung V, Endres S, Ruzicka T, Rothenfusser S and Hartmann G: Proapoptotic signaling induced by RIG-I and MDA-5 results in type I interferon-independent apoptosis in human melanoma cells. J Clin Invest 119: 2399-2411, 2009.

8. Yuan D, Xia M, Meng G, Xu C, Song Y and Wei J: Anti-angiogenic efficacy of 5'-triphosphate siRNA combining VEGF silencing and RIG-I activation in NSCLCs. Oncotarget 6: 29664-29674, 2015.

9. Yoshino H, Chiba K, Saitoh T and Kashiwakura I: Ionizing radiation affects the expression of Toll-like receptors 2 and 4 in human monocytic cells through c-Jun $\mathrm{N}$-terminal kinase activation. J Radiat Res 55: 876-884, 2014.

10. Yoshino H, Saitoh T, Kozakai M and Kashiwakura I: Effects of ionizing radiation on retinoic acid-inducible gene-I-like receptors. Biomed Rep 3: 59-62, 2015.

11. Tsai MF, Wang CC and Chen JJ: Tumour suppressor HLJ1: A potential diagnostic, preventive and therapeutic target in non-small cell lung cancer. World J Clin Oncol 5: 865-873, 2014

12. Yoshino H, Kumai Y and Kashiwakura I: Effects of endoplasmic reticulum stress on apoptosis induction in radioresistant macrophages. Mol Med Rep 15: 2867-2872, 2017.

13. Fukushi S, Yoshino H, Yoshizawa A and Kashiwakura I: p53-independent structure-activity relationships of 3-ring mesogenic compounds' activity as cytotoxic effects against human non-small cell lung cancer lines. BMC Cancer 16: 521, 2016.

14. Rogakou EP, Pilch DR, Orr AH, Ivanova VS and Bonner WM: DNA double-stranded breaks induce histone H2AX phosphorylation on serine 139. J Biol Chem 273: 5858-5868, 1998.

15. Jacobs JL and Coyne CB: Mechanisms of MAVS regulation at the mitochondrial membrane. J Mol Biol 425: 5009-5019, 2013.
16. Castanier C, Zemirli N, Portier A, Garcin D, Bidère N, Vazquez A and Arnoult D: MAVS ubiquitination by the E3 ligase TRIM25 and degradation by the proteasome is involved in type I interferon production after activation of the antiviral RIG-I-like receptors. BMC Biol 10: 44, 2012.

17. Imaizumi $\mathrm{T}$, Aizawa-Yashiro $\mathrm{T}$, Tsuruga $\mathrm{K}$, Tanaka $\mathrm{H}$, Matsumiya T, Yoshida H, Tatsuta T, Xing F, Hayakari R and Satoh K: Melanoma differentiation-associated gene 5 regulates the expression of a chemokine CXCL10 in human mesangial cells: Implications for chronic inflammatory renal diseases. Tohoku J Exp Med 228: 17-26, 2012.

18. Poeck H, Besch R, Maihoefer C, Renn M, Tormo D, Morskaya SS, Kirschnek S, Gaffal E, Landsberg J, Hellmuth J, et al: 5'-Triphosphate-siRNA: Turning gene silencing and Rig-Iactivation against melanoma. Nat Med 14: 1256-1263, 2008.

19. Li D, Gale RP, Liu Y, Lei B, Wang Y, Diao D and Zhang M: 5'-Triphosphate siRNA targeting MDR1 reverses multi-drug resistance and activates RIG-I-induced immune-stimulatory and apoptotic effects against human myeloid leukaemia cells. Leuk Res 58: 23-30, 2017.

20. Duewell P, Steger A, Lohr H, Bourhis H, Hoelz H, Kirchleitner SV, Stieg MR, Grassmann S, Kobold S, Siveke JT, et al: RIG-I-like helicases induce immunogenic cell death of pancreatic cancer cells and sensitize tumors toward killing by CD8(+) T cells. Cell Death Differ 21: 1825-1837, 2014.

21. Ranoa DR, Parekh AD, Pitroda SP, Huang X, Darga T, Wong AC, Huang L, Andrade J, Staley JP, Satoh T, et al: Cancer therapies activate RIG-I-like receptor pathway through endogenous non-coding RNAs. Oncotarget 7: 26496-26515, 2016.

22. Widau RC, Parekh AD, Ranck MC, Golden DW, Kumar KA, Sood RF, Pitroda SP, Liao Z, Huang X, Darga TE, et al: RIG-I-like receptor LGP2 protects tumor cells from ionizing radiation. Proc Natl Acad Sci USA 111: E484-E491, 2014.

23. Burnette BC, Liang H, Lee Y, Chlewicki L, Khodarev NN, Weichselbaum RR, Fu YX and Auh SL: The efficacy of radiotherapy relies upon induction of type i interferon-dependent innate and adaptive immunity. Cancer Res 71: 2488-2496, 2011.

24. Schmidberger H, Rave-Fränk M, Lehmann J, Schweinfurth S, Rehring E, Henckel K and Hess CF: The combined effect of interferon beta and radiation on five human tumor cell lines and embryonal lung fibroblasts. Int J Radiat Oncol Biol Phys 43: 405-412, 1999.

25. Lei Y, Moore CB, Liesman RM, O'Connor BP, Bergstralh DT, Chen ZJ, Pickles RJ and Ting JP: MAVS-mediated apoptosis and its inhibition by viral proteins. PLoS One 4: e5466, 2009.

26. Yu CY, Chiang RL, Chang TH, Liao CL and Lin YL: The interferon stimulator mitochondrial antiviral signaling protein facilitates cell death by disrupting the mitochondrial membrane potential and by activating caspases. J Virol 84: 2421-2431, 2010.

27. El Maadidi S, Faletti L, Berg B, Wenzl C, Wieland K, Chen ZJ, Maurer U and Borner C: A novel mitochondrial MAVS/Caspase-8 platform links RNA virus-induced innate antiviral signaling to Bax/Bak-independent apoptosis. J Immunol 192: 1171-1183, 2014.

28. Verbrugge I, de Vries E, Tait SW, Wissink EH, Walczak H, Verheij $\mathrm{M}$ and Borst J: Ionizing radiation modulates the TRAIL death-inducing signaling complex, allowing bypass of the mitochondrial apoptosis pathway. Oncogene 27: 574-584, 2008.

29. Kim MJ, Lee KH and Lee SJ: Ionizing radiation utilizes c-Jun $\mathrm{N}$-terminal kinase for amplification of mitochondrial apoptotic cell death in human cervical cancer cells. FEBS J 275: 2096-2108, 2008.

30. Maier P, Hartmann L, Wenz F and Herskind C: Cellular pathways in response to ionizing radiation and their targetability for tumor radiosensitization. Int J Mol Sci 17: pii: E102, 2016.

31. Kim JY, An YM, Choi WH, Kim JM, Cho S, Yoo BR, Kang JW, Lee YS, Lee YJ and Cho J: Pro-apoptotic Noxa is involved in ablative focal irradiation-induced lung injury. J Cell Mol Med 21: 711-719, 2017.

32. Liu J, Guo YM, Hirokawa M, Iwamoto K, Ubukawa K, Michishita Y, Fujishima N, Tagawa H, Takahashi N, Xiao W, et al: A synthetic double-stranded RNA, poly I:C, induces a rapid apoptosis of human CD34(+) cells. Exp Hematol 40: 330-341, 2012. 